\title{
Cooperative Transmission with Partial Channel Information in Multi-User MISO Wireless Systems
}

\author{
Keon-Wook Lee and Yong-Hwan Lee \\ School of Electrical Engineering and INMC, Seoul National University \\ Kwanak P. O. Box 34, Seoul, 151-600 Korea
}

\begin{abstract}
In this paper, we consider novel cooperative transmission from multiple base stations in multi-user wireless systems. The proposed scheme generates multiple beams using partial channel state information, enabling to achieve both beamforming and spatial multiplexing gain. With the use of twophase multi-user scheduling, it can also achieve multi-user diversity gain. The performance of the proposed scheme is analyzed and verified by computer simulation. Simulation results show the proposed scheme is quite effective as the spatial correlation and/or the number of users increase.
\end{abstract}

\section{INTRODUCTION}

The use of multiple antennas, so called multi-input multioutput (MIMO), has recently received attention as a key technology for future wireless communications. When the channel gain between the transmit and receive antennas is independent and identically distributed (i.i.d.), the channel capacity increases linearly proportional to the minimum number of transmit and receive antennas [1], [2]. However, the capacity of a cellular system near the cell boundary is mainly limited by interference from other cells, so-called other-cell interference (OCI) [3]. Even when the user receives the signal with a good quality from the serving cell, it may not properly communicate with its serving cell due to large interference from adjacent cells. In fact, it is required not only to maximize the receive power of the desired signal, but also to minimize the interference from adjacent $\mathrm{c}$ ells.

The use of a centralized scheduler has recently been considered to mitigate the OCI problem. A centralized scheduler installed at the top of a cluster comprising several BSs can maximize the overall system performance by jointly considering the cell loading and channel state information (CSI) of all users [4]. Besides, it is possible to employ cooperation among multiple BSs, which enables users to receive useful signal rather than interference from adjacent BSs, improving the performance near the cell boundary.

A few numbers of studies have recently been reported on cooperative transmission in a cellular downlink system. Treating multiple BSs as a single giant BS, the sum rate capacity is maximized by applying dirty paper coding (DPC) [5] in the MIMO downlink [6], [7]. It has been shown that the sum rate capacity can be achieved asymptotically by means of

This work was partly supported by the IT R\&D program of MKE/IITA [2008-F-007-01, Wireless Communication Systems in 3 Dimensional Environment] and Seoul R\&BD Program (10544). cooperation of BSs with the use of a zero-forcing DPC (ZFDPC) scheme [8], [9]. However, the use of DPC requires huge processing complexity and full CSI at the transmitter, making it impractical for realization. The use of linear precoding based joint $\mathrm{ZF}$ transmission has been considered to reduce the complexity [10], [11]. However, it also requires full CSI at the transmitter and requires the searching complexity increasing prohibitively large as the number of users increases [12].

To improve the performance near the cell boundary, we consider the use of cooperative transmission in the centralized scheduler architecture. To reduce the implementation complexity and feedback signaling overhead, the proposed scheme generates multiple beams using partial CSI such as the spatial channel covariance information, phase information for co-phase reception and signal to interference power ratio (SINR) with the beam index. The proposed scheme can simultaneously provide the beamforming and spatial multiplexing gain as well as the multi-user diversity gain, by generating beams in a two-step process. It generates the first random beam to achieve beamforming gain. Then, it generates the next random beam which is orthogonal to the first beam to achieve spatial multiplexing gain and multi-user diversity gain.

The rest of the paper is organized as follows. Section II describes the system model and Section III describes the proposed BS cooperative transmission scheme. The performance of the proposed scheme is analyzed in Section IV and verified by computer simulation in Section V. Finally, Section VI summarizes conclusions.

\section{SYSTEM MODEL}

Consider the downlink of a multi-cell multi-user wireless system comprising $N_{B S}$ BSs, where each BS transmits the signal using $M$ transmit antennas with beamforming and each of $N$ users receives it using a single receive antenna. We assume that users estimate the CSI from a common pilot signal and then report it to the BS through an uplink feedback channel. We also assume that all the BSs are synchronized to each other with universal frequency reuse and connected to a centralized scheduler via a high-speed backbone network for reliable and rapid exchange of information.

For given signal transmitted from $N_{B S} \mathrm{BSs}$, the received signal $y_{k}$ of user $k$ can be represented as

$$
y_{k}=\sum_{i=1}^{N_{\text {BS }}} a_{i, k} \mathbf{h}_{i, k}^{*} \mathbf{w}_{i, k} s_{k}+\sum_{i=1}^{N_{\text {BS }}} \sum_{j \neq k} a_{i, k} \mathbf{h}_{i, k}^{*} \mathbf{w}_{i, j} s_{j}+n_{k},
$$


where $a_{i, k}$ and $\mathbf{h}_{i, k}$ respectively denote the large-scale fading coefficient and the $(M \times 1)$ small-scale fading channel vector from BS $i$ to user $k, \mathbf{w}_{i, k}$ and $s_{k}$ respectively denote the $(M \times 1)$ beam weight vector and data symbol of BS $i$ for user $k$, and $n_{k}$ represents additive white Gaussian noise (AWGN) with variance $N_{0}$. Here, the superscript $*$ denotes conjugate transpose. Without loss of generality, we assume that all the BSs have the same average transmit power $P_{T}$. To preserve the transmit power constraint, $\left\|\mathbf{w}_{i, k}\right\|$ is set to one for all $i$ and $k$, where $\|\mathbf{w}\|$ denotes the Frobenius norm of $\mathbf{w}$.

In a spatially correlated Rayleigh fading channel environment, $\mathbf{h}_{i, k}$ can be represented as [14]

$$
\mathbf{h}_{i, k}=\mathbf{R}_{i, k}^{1 / 2} \tilde{\mathbf{h}}_{i, k},
$$

where $\tilde{\mathbf{h}}_{i, k}$ denotes the $(M \times 1)$ uncorrelated virtual channel vector from BS $i$ to user $k$, whose elements are i.i.d. zeromean complex Gaussian random variables with unit variance, and $\mathbf{R}_{i, k}$ denotes the $(M \times M)$ spatial channel covariance matrix defined by

$$
\mathbf{R}_{i, k}=E\left\{\mathbf{h}_{i, k} \mathbf{h}_{i, k}^{*}\right\} .
$$

Since $\mathbf{R}_{i, k}$ is a Hermitian matrix, it can be represented as

$$
\mathbf{R}_{i, k}=\mathbf{V}_{i, k} \boldsymbol{\Sigma}_{i, k} \mathbf{V}_{i, k}^{*}
$$

where $\quad \mathbf{V}_{i, k}=\left[\begin{array}{lll}\mathbf{v}_{i, k}^{(1)} & \cdots & \mathbf{v}_{i, k}^{(M)}\end{array}\right]$ is an $(M \times M)$ unitary matrix whose columns are the normalized eigenvectors of $\mathbf{R}_{i, k}$ and $\boldsymbol{\Sigma}_{i, k}$ is an $(M \times M)$ diagonal matrix whose diagonal elements $\left\{\lambda_{i, k}^{(1)}, \cdots, \lambda_{i, k}^{(M)}\right\}$ are descending ordered non-negative real values, i.e., $\quad \lambda_{i, k}^{(1)} \geq \lambda_{i, k}^{(2)} \cdots \geq \lambda_{i, k}^{(M)} \geq 0$. We define the principle eigenvector by $\mathbf{v}_{i, k}^{\max }$ (i.e., $\mathbf{v}_{i, k}^{(1)}=\mathbf{v}_{i, k}^{\max }$ ).

\section{Proposed CoOperative TRANSMISSION SCHEME}

We consider the use of two-phase scheduling as illustrated in Fig. 1. In the first scheduling phase, centralized scheduler selects a user achieving the maximum beamforming gain based on the spatial channel covariance matrix, without the use of instantaneous channel matrix. To do this, all users need to report the instantaneous SINR to the centralized scheduler by assuming that all the cooperating BSs serve the data based on their own principle eigenvector. Since the spatial channel covariance matrix is not changed for a relatively long duration compared to the instantaneous channel state, it doesn't have to be reported very often. Assuming no correlation between the antennas in other BSs, it can be possible for each BS to determine the beam weight vector without considering the channel covariance information of other BSs. Thus, (1) can be rewritten as

$$
y_{k}=\sum_{i=1}^{N_{\mathrm{BS}}} a_{i, k} \mathbf{h}_{i, k}^{*} \mathbf{v}_{i, k}^{\max } s_{k}+\sum_{i=1}^{N_{\mathrm{BS}}} \sum_{j \neq k}^{M} a_{i, k} \mathbf{h}_{i, k}^{*} \mathbf{w}_{i, j} S_{j}+n_{k} .
$$

Further performance improvement can be achieved by means of co-phase reception of the desired signal. To this end,

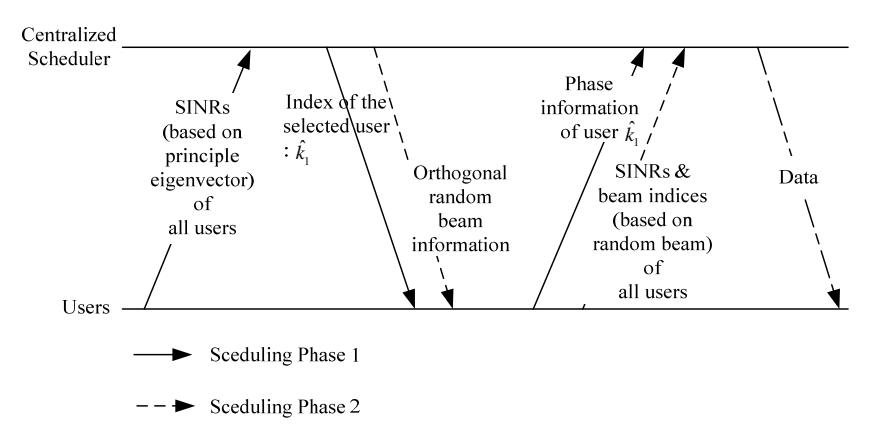

Fig. 1. Procedure of proposed BS cooperative transmission scheme.

assume that $\mathbf{v}_{i, k}^{\max }$ is rotated by $\theta_{i, k}$, where

$$
\theta_{i, k}= \begin{cases}0, & \text { for } i=1 \\ \measuredangle \mathbf{h}_{1, k}^{*} \mathbf{v}_{1, k}^{\max }-\measuredangle \mathbf{h}_{i, k}^{*} \mathbf{v}_{i, k}^{\max }, & \text { otherwise. }\end{cases}
$$

Then, (5) can be rewritten as

$$
\begin{aligned}
y_{k} & =\sum_{i=1}^{N_{B S}} a_{i, k} \mathbf{h}_{i, k}^{*} \mathbf{v}_{i, k}^{\max } e^{\mathrm{i} \cdot \theta_{i, k}} s_{k}+\sum_{i=1}^{N_{\text {BS }}} \sum_{j \neq k}^{M} a_{i, k} \mathbf{h}_{i, k}^{*} \mathbf{w}_{i, j} e^{\mathrm{i} \cdot \theta_{i, j}} s_{j}+n_{k} \\
& =\sum_{i=1}^{N_{B S}}\left|a_{i, k} \mathbf{h}_{i, k}^{*} \mathbf{v}_{i, k}^{\max } s_{k}\right|+\sum_{i=1}^{N_{B S}} \sum_{j \neq k}^{M} a_{i, k} \mathbf{h}_{i, k}^{*} \mathbf{w}_{i, j} s_{j}+n_{k},
\end{aligned}
$$

where $\mathrm{i}=\sqrt{-1}$. Thus, in the first scheduling phase, user $k$ needs to report the instantaneous SINR $\gamma_{k}$ to the centralized scheduler, represented as

$$
\gamma_{k}=\frac{\left(\sum_{i=1}^{N_{\text {BS }}}\left|a_{i, k} \mathbf{h}_{i, k}^{*} \mathbf{v}_{i, k}^{\max } \sqrt{\frac{P_{T}}{M}}\right|\right)^{2}}{\sum_{i=1}^{N_{\text {BS }}} \sum_{j \neq k}^{M} a_{i, k}^{2}\left|\mathbf{h}_{i, k}^{*} \mathbf{w}_{i, j}\right|^{2} \frac{P_{T}}{M}+N_{0}} .
$$

Since $\left\{\mathbf{w}_{i, j} ; i=1, \cdots, N_{B S}, j \neq k\right\}$ are not determined, it may not be possible for user $k$ to calculate $\gamma_{k}$ in (8). However, when the proposed scheme randomly generate $\mathbf{w}_{i, j}$ orthogonal to $\mathbf{v}_{i, k}^{\max }$, yielding less interference to user , (8) can uniquely be determined by

$$
\gamma_{k}=\frac{\left(\sum_{i=1}^{N_{\mathrm{BS}}} \mid a_{i, k} \mathbf{h}_{i, k}^{*} \mathbf{v}_{i, k}^{\max } \sqrt{\left.\frac{P_{T}}{M} \mid\right)^{2}}\right.}{\sum_{i=1}^{N_{\mathrm{BS}}} \sum_{j \neq 1}^{M} a_{i, k}^{2}\left|\mathbf{h}_{i, k}^{*} \mathbf{v}_{i, k}^{(j)}\right|^{2} \frac{P_{T}}{M}+N_{0}} .
$$

Then, the centralized scheduler selects a best user $\hat{k}_{1}$ from $\left\{\gamma_{k} ; k=1, \cdots, N\right\}$ and the selected user $\hat{k}_{1}$ reports the phase information $\left\{\theta_{i, k} ; i=2, \cdots, N_{B S}\right\}$ to the BS.

After the first scheduling phase, the proposed scheme generates multiple beams to obtain multiplexing gain. For random beams $\left\{\tilde{\mathbf{w}}_{i, j} ; i=1, \cdots, N_{B S}, j=1, \cdots, M-1\right\}$ orthogonal to $\mathbf{v}_{i, \hat{k}_{1}}^{\max }$, all users report their highest instantaneous SINR with the corresponding beam index. In the second scheduling phase, user $k$ needs to report the instantaneous SINR $\gamma_{k}^{\prime}$ to the 
centralized scheduler, represented as

$$
\gamma_{k}^{\prime}=\arg \max _{j=1, \cdots, M-1} \frac{\sum_{i=1}^{N_{B S}} a_{i, k}^{2}\left|\mathbf{h}_{i, k}^{*} \tilde{\mathbf{w}}_{i, j}\right|^{2} \frac{P_{T}}{M}}{\sum_{i=1}^{N_{B S}} \sum_{m \neq j}^{M} a_{i, k}^{2}\left|\mathbf{h}_{i, k}^{*} \tilde{\mathbf{w}}_{i, m}\right|^{2} \frac{P_{T}}{M}+N_{0}} .
$$

Then, the centralized scheduler selects a user based on the instantaneous SINR $\left\{\gamma_{k}^{\prime}\right\}$ and transmits the data with the corresponding beam weight.

\section{PERFORMANCE ANALYSIS}

For analysis of the performance of the proposed scheme, First, consider the performance due to the first scheduling phase. When the centralized scheduler assigns the resource to a user having the highest SINR, the selected user index $\hat{k}_{1}$ can be represented as

$$
\hat{k}_{1} \triangleq \arg \max _{k=1, \cdots, N}\left\{\gamma_{k}\right\}
$$

In (2), $\tilde{\mathbf{h}}_{i, k}$ can be represented using orthonormal bases $\left\{\mathbf{O}_{i, k}^{(1)}, \mathbf{O}_{i, k}^{(2)}, \cdots, \mathbf{O}_{i, k}^{(M)} \in \mathbb{C}^{M \times 1}\right\}$ as

$$
\tilde{\mathbf{h}}_{i, k}=\alpha_{i, k}^{(1)} \mathbf{O}_{i, k}^{(1)}+\alpha_{i, k}^{(2)} \mathbf{O}_{i, k}^{(2)}+\cdots+\alpha_{i, k}^{(M)} \mathbf{O}_{i, k}^{(M)} .
$$

Since the elements of $\tilde{\mathbf{h}}_{i, k}$ are complex Gaussian random variables, $\left\{\alpha_{i, k}^{(1)}, \cdots, \alpha_{i, k}^{(M)}\right\}$ are also i.i.d. zero mean complex Gaussian random variables with unit variance. Letting $\mathbf{V}_{i, k}$ be an orthonormal basis (i.e., $\mathbf{O}_{i, k}^{(j)}=\mathbf{v}_{i, k}^{(j)}$ for $\left.j=1, \cdots M\right)$ ), it can be shown that

$$
\left\|\mathbf{h}_{i, k}\right\|=\sum_{j=1}^{M}\left|\mathbf{h}_{i, k}^{*} \mathbf{v}_{i, k}^{(j)}\right|^{2}=\sum_{j=1}^{M}\left|\tilde{\mathbf{h}}_{i, k}^{*} \mathbf{R}_{i, k}^{1 / 2} \mathbf{v}_{i, k}^{(j)}\right|^{2}=\sum_{j=1}^{M} \lambda_{i, k}^{(j)}\left|\alpha_{i, k}^{(j)}\right|^{2} .
$$

Then, the corresponding SINR of user $\hat{k}_{1}$ can be represented as

$$
\gamma_{\hat{k}_{1}} \triangleq \max _{k=1, \cdots, N}\left\{\frac{\left(\sum_{i=1}^{N_{B S}}\left|a_{i, k} \alpha_{i, k}^{(1)} \sqrt{\frac{\lambda_{i, k}^{(1)} P_{T}}{M}}\right|\right)^{2}}{\sum_{i=1}^{N_{B S}} \sum_{j=2}^{M} a_{i, k}^{2} \lambda_{i, k}^{(j)}\left|\alpha_{i, k}^{(j)}\right|^{2} \frac{P_{T}}{M}+N_{0}}\right\} .
$$

Note that when the spatial correlation is high, most of the channel power is concentrated on $\lambda_{i, k}^{(1)}$, yielding a small value of $\sum_{j=2}^{M} \lambda_{i, k}^{(j)}=M-\lambda_{i, k}^{(1)}$. Thus, the co-channel interference term (i.e., the first term in the denominator of (14)) can also be approximated as its average power. Then (14) can further be approximated as

$$
\gamma_{\hat{k}_{1}} \approx \max _{k=1, \cdots, N}\left\{\left(\sum_{i=1}^{N_{\mathrm{BS}}}\left|\frac{a_{i, k} \alpha_{i, k}^{(1)}}{\sqrt{I_{k}}} \sqrt{\frac{\lambda_{i, k}^{(1)} P_{T}}{M}}\right|\right)^{2}\right\},
$$

where $I_{k}=\sum_{i=1}^{N_{B S}} \sum_{j=2}^{M} a_{i, k}^{2} \lambda_{i, k}^{(j)}\left|\alpha_{i, k}^{(j)}\right|^{2} P_{T} / M+N_{0}$.

It can be seen from (15) that $\gamma_{\hat{k}_{1}}$ is the maximum of a squared sum of independent Rayleigh random variables having different mean values, which is not easily analyzable [16], [17]. Instead, it can be shown from an inequality of the arithmetic and geometric means that the term in (15) is bounded as

$$
\begin{aligned}
& \left(\sum_{i=1}^{N_{\mathrm{BS}}}\left|\frac{a_{i, k} \alpha_{i, k}^{(1)}}{\sqrt{I_{k}}} \sqrt{\frac{\lambda_{i, k}^{(1)} P_{T}}{M}}\right|\right)^{2} \\
& \leq \frac{1}{2} \sum_{i=1}^{N_{B S}} \sum_{j=1}^{N_{B S}} \frac{a_{i, k} a_{j, k}}{I_{k}} \sqrt{\frac{\lambda_{i, k}^{(1)} P_{T}}{M}} \sqrt{\frac{\lambda_{j, k}^{(1)} P_{T}}{M}}\left(\left|\alpha_{i, k}^{(1)}\right|^{2}+\left|\alpha_{j, k}^{(1)}\right|^{2}\right) \\
& =\sum_{i=1}^{N_{B S}} A_{i, k}\left|\alpha_{i, k}^{(1)}\right|^{2} \text {, }
\end{aligned}
$$

where $A_{i, k}=\sum_{j=1}^{N_{\mathrm{BS}}}\left(a_{i, k} a_{j, k} / I_{k} \sqrt{\lambda_{i, k}^{(1)} P_{T} / M} \sqrt{\lambda_{j, k}^{(1)} P_{T} / M}\right)$. In practice, however, two or three BSs are involved for cooperative communications mainly due to the cellular geometry. Then, $\gamma_{\hat{k}_{1}}$ can be approximated as

$$
\gamma_{\hat{k}_{1}} \approx \max _{k=1, \cdots, N}\left\{\sum_{i=1}^{N_{B S}} A_{i, k}\left|\alpha_{i, k}^{(1)}\right|^{2}\right\} .
$$

Letting $\quad \tilde{\gamma}_{k} \triangleq \sum_{i=1}^{N_{\mathrm{BS}}} A_{i, k}\left|\alpha_{i, k}^{(1)}\right|^{2}, \quad \tilde{\gamma}_{k}$ is a sum of independent exponential random variables with different mean values. Thus, the pdf and the cumulative density function (cdf) of $\tilde{\gamma}_{k}$ are respectively given by [15]

$$
\begin{gathered}
f_{\tilde{\gamma}_{k}}(z)=\sum_{i=1}^{N_{\mathrm{BS}}} \frac{1}{A_{i, k}} \prod_{j \neq i}^{N_{\mathrm{BS}}} \frac{A_{i, k}}{A_{i, k}-A_{j, k}} e^{-\frac{z}{A_{i, k}}}=\sum_{i=1}^{N_{\mathrm{BS}}} B_{i, k} e^{-\frac{z}{A_{i, k}}}, \\
F_{\tilde{\gamma}_{k}}(z)=\int_{0}^{z} B_{i, k} e^{-\frac{z}{A_{i, k}}} d z=\sum_{i=1}^{N_{\mathrm{BS}}} A_{i, k} B_{i, k}\left(1-e^{-\frac{z}{A_{i, k}}}\right),
\end{gathered}
$$

where $B_{i, k}=\left(\prod_{j \neq i}^{N_{B S}} A_{i, k} /\left(A_{i, k}-A_{j, k}\right)\right) / A_{i, k}$. Since $\left\{\tilde{\gamma}_{1}, \tilde{\gamma}_{2}, \cdots, \tilde{\gamma}_{N}\right\}$ are independent of each other, the cdf and pdf of $\gamma_{\hat{k}_{1}}$ can respectively be represented as

$$
\begin{gathered}
F_{\gamma_{\hat{k}_{1}}}(z)=\operatorname{Pr}\left\{\gamma_{\hat{k}_{1}} \leq z\right\}=F_{\tilde{\gamma}_{1}}(z) \cdot F_{\tilde{\gamma}_{2}}(z) \cdots F_{\tilde{\gamma}_{N}}(z) \\
=\prod_{k=1}^{N}\left(\sum_{i=1}^{N_{B S}} A_{i, k} B_{i, k}\left(1-e^{-\frac{z}{\hat{A}_{i, k}}}\right)\right), \\
f_{\gamma_{\hat{k}_{1}}}(z)=\left[\prod_{k=1}^{N} F_{\tilde{\gamma}_{k}}(z)\right] \cdot \sum_{k=1}^{N_{u}} \frac{f_{\tilde{\gamma}_{k}}(z)}{F_{\tilde{\gamma}_{k}}(z)}=\sum_{k=1}^{N}\left[f_{\tilde{\gamma}_{k}}(z) \cdot \prod_{j=1, j \neq k}^{N} F_{\tilde{\gamma}_{j}}(z)\right] \\
=\sum_{k=1}^{N} \sum_{i=1}^{N_{B S}} B_{i, k}\left(e^{-\frac{z}{A_{i, k}}} \cdot \prod_{j=1, j \neq k}^{N}\left(\sum_{l=1}^{N_{B S}} A_{l, j} B_{l, j}\left(1-e^{-\frac{z}{A_{l, j}}}\right)\right)\right)
\end{gathered}
$$

It can be shown after some manipulations that (21) can be represented in a closed form as 


$$
\begin{aligned}
f_{\gamma_{k_{1}}}(z)= & \sum_{i=1}^{N} \sum_{j=1}^{N C_{i}}\left[\sum_{l=1}^{\left(N_{\mathrm{BS}}\right)^{i}}(-1)^{1+i}\left(\prod_{k=1, k \notin \Lambda_{i, j}}^{N}\left(\sum_{l=1}^{N_{\mathrm{BS}}} A_{l, k} B_{l, k}\right)\right)\right. \\
& \cdot\left\{\left(\prod_{k=1}^{i} A_{\Phi_{\Phi_{i}}(k, l), \Lambda_{i}(k, j)} B_{\Phi_{i}(k, l), \Lambda_{i}(k, j)}\right) \cdot e^{-\left(\sum_{k=1}^{i} \frac{1}{\left.A_{\Phi_{i}(k, l), \Lambda_{i}(k, j)}\right)}\right.}\right. \\
& \left.\left.\cdot\left(\sum_{k=1}^{i} \frac{1}{A_{\Phi_{i}(k, l), \Lambda_{i}(k, j)}}\right)\right\}\right]
\end{aligned}
$$

where $\Phi_{i}$ denotes an $\left(i \times\left(N_{B S}\right)^{i}\right)$ matrix whose columns are the outcomes of $i$-permutation with repetition from the $N_{B S}$ BS index set, $\Lambda_{i}$ denotes an $\left(i \times{ }_{N} C_{i}\right)$ matrix whose columns are the outcomes of $i$-combination without repetition from the $N$-user index set, $\Lambda_{i, j}$ denotes the $j$-th column of $\Lambda_{i}$, and $\Phi_{i}(k, l)$ and $\Lambda_{i}(k, l)$ denote the element of the $k$-th row and the $l$-th column of $\Phi_{i}$ and $\Lambda_{i}$, respectively. Here ${ }_{N} C_{i}$ denotes the number of $i$-combinations without repetition form a set of $N$ elements. For example, when $N_{B S}=2$ and $N=3$, $\Phi_{2}$ and $\Lambda_{2}$ can be represented as respectively,

$$
\begin{gathered}
\Phi_{2}=\left[\begin{array}{llll}
1 & 1 & 2 & 2 \\
1 & 2 & 1 & 2
\end{array}\right], \\
\Lambda_{2}=\left[\begin{array}{lll}
1 & 1 & 2 \\
2 & 3 & 3
\end{array}\right] .
\end{gathered}
$$

Thus, the channel capacity of user $\hat{k}_{1}$ can be represented as

$$
\begin{aligned}
C_{\hat{k}_{1}}= & \int_{0}^{\infty} \log _{2}(1+z) \cdot f_{\gamma_{\hat{k}_{1}}}(z) d z \\
= & \frac{1}{\ln 2} \sum_{i=1}^{N} \sum_{j=1}^{N} C_{i}\left[\sum_{l=1}^{\left(N_{B S}\right)^{i}}(-1)^{1+i} \cdot E_{i}\left(\sum_{k=1}^{i} \frac{1}{A_{\Phi_{i}(k, l), \Lambda_{i}(k, j)}}\right) \cdot e^{\sum_{k=1}^{i} \frac{1}{A_{b_{i}(k, l), \Lambda_{i}(k, j)}}}\right. \\
& \left.\cdot\left(\prod_{k=1}^{i} A_{\Phi_{i}(k, l), \Lambda_{i}(k, j)} B_{\Phi_{i}(k, l), \Lambda_{i}(k, j)}\right) \cdot\left(\prod_{k=1, k \notin \Lambda_{i, j}}^{N}\left(\sum_{l=1}^{N_{B S}} A_{l, k} B_{l, k}\right)\right)\right],
\end{aligned}
$$

where $E_{i}(z)=\int_{z}^{\infty} e^{-t} / t d t$.

The performance of the second phase scheduling can similarly be analyzed. Assuming that the selected user index of the $m$-th beam is $\left\{\hat{k}_{m} ; m=2, \ldots, M\right\}$. Since second phase scheduling can not exploit beamforming gain, corresponding channel capacity $C_{\hat{k}_{m}}$ of user $\hat{k}_{m}$ can be represented as

$$
\begin{aligned}
C_{\hat{k}_{m}} & =\frac{1}{\ln 2} \sum_{i=1}^{N} \sum_{j=1}^{N} C_{i}\left[\sum_{l=1}^{\left(N_{B S}\right)^{i}}(-1)^{1+i} E_{i}\left(\sum_{k=1}^{i} \frac{1}{A_{\Phi_{i}(k, l), \Lambda_{i}(k, j)}^{\prime}}\right) e^{\sum_{k=1}^{i}} \frac{1}{A_{\Phi_{i}(k, l), \Lambda_{i}(k, j)}^{\prime}}\right. \\
& \left.\cdot\left(\prod_{k=1}^{i} A_{\Phi_{i}(k, l), \Lambda_{i}(k, j)}^{\prime} B_{\Phi_{i}(k, l), \Lambda_{i}(k, j)}^{\prime}\right) \cdot\left(\prod_{k=1, k \notin \Lambda_{i, j}}^{N}\left(\sum_{l=1}^{N_{B S}} A_{l, k}^{\prime} B_{l, k}^{\prime}\right)\right)\right],
\end{aligned}
$$

where $A_{i, k}^{\prime}=a_{i, k}^{2} P_{T} /\left(I_{k} M\right)$ and $B_{i, k}^{\prime}=\left(\prod_{j \neq i}^{N_{\mathrm{BS}}} A_{i, k}^{\prime} /\left(A_{i, k}^{\prime}-A_{j, k}^{\prime}\right)\right) / A_{i, k}^{\prime}$. Finally, since $\left\{\hat{k}_{m} ; m=2, \ldots, M\right\}$ have the same statistical characteristics, the channel capacity of the proposed scheme
TABLE I.

SIMULATION PARAMETERS

\begin{tabular}{|c|c|}
\hline \multicolumn{2}{|c|}{ SIMULATION PARAMETERS } \\
\hline Cell configuration & 27 cells \\
\hline Cell radius & $1 \mathrm{~km}$ \\
\hline Carrier frequency & $5.8 \mathrm{GHz}$ \\
\hline Symbol time & $25.48 \mu \mathrm{s}$ \\
\hline Feedback delay & 64 symbol \\
\hline Antenna configuration & $4 \times 1$ \\
\hline Path-loss exponent & 4 \\
\hline Link adaptation & Ideal (i.e., using the Shannon's \\
& capacity formula) \\
\hline
\end{tabular}

can be represented as

$$
C_{N_{B S}, N}=\sum_{i=1}^{M} C_{\hat{k}_{i}}=C_{\hat{k}_{1}}+(M-1) C_{\hat{k}_{2}} .
$$

\section{Simulation Results}

The performance of the proposed scheme is verified assuming the use of two cooperating BSs in a two-tiered hexagonal shaped cellular network (i.e., 27 cells) by computer simulation. We consider the use of a $(4 \times 1)$ antenna configuration for the signal transmission over a spatially correlated Rayleigh fading channel with complex-valued spatial correlation coefficient $\rho$ between the adjacent antennas. We assume that all concerning users are located in the common boundary of two center cells and experience the same correlation magnitude $|\rho|$. The simulation parameters are summarized in TABLE I [18]. For fair comparison, the performance of the proposed scheme is compared with two reference schemes with a same multiplexing order of 4; linear joint ZF beamforming based on full CSI as a conventional cooperative transmission scheme [10], [11] and orthogonal random beamforing based on partial CSI (i.e., the best beam index with the corresponding SINR) as a non-cooperative one [13]. Notice that the proposed scheme employs a two-phase scheduling scheme as in Fig. 1, taking a time delay twice that of the reference schemes for the generation of the first beam.

Fig. 2 depicts the spectral efficiency in terms of the number of users distributed near the boundary of two cells with a value of $|\rho|=0.01$ or $|\rho|=0.99$ at a mobility of $0 \mathrm{~km}$ (i.e., no feedback delay) or $60 \mathrm{~km}$. The time correlation of the channel is generated by the Jake's model [19]. It can be seen that the performance of the linear joint ZF beamforming outperforms the other schemes in the presence of no mobility. The use of full CSI with no feedback delay enables the linear joint ZF beamforming to perfectly eliminate the CCI by cooperative joint transmission. Moreover, it can achieve multi-user diversity gain by allocating the resource to a user in the best channel condition. On the other hand, the orthogonal random beamforing achieves multi-user diversity gain but suffers from OCI due to no cooperation with adjacent cells, providing the worst performance among the three schemes. When $|\rho|=0.01$, 


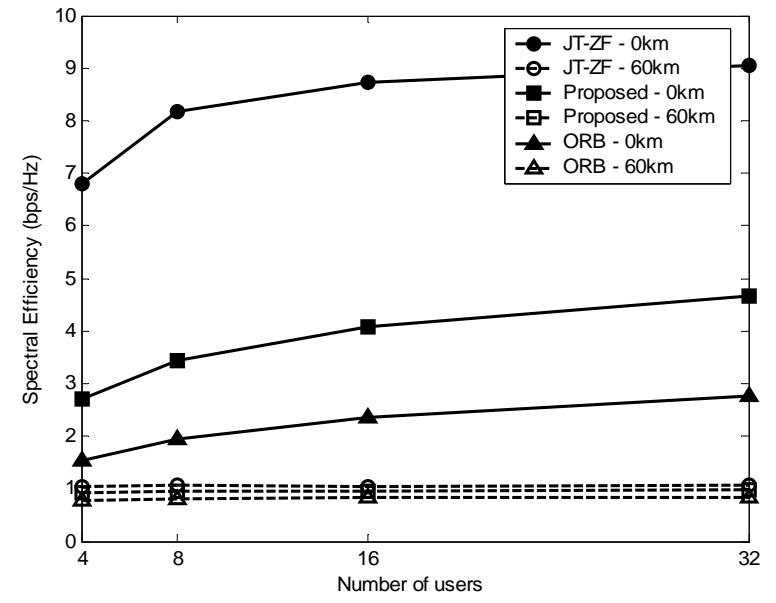

(a) $|\rho|=0.01$

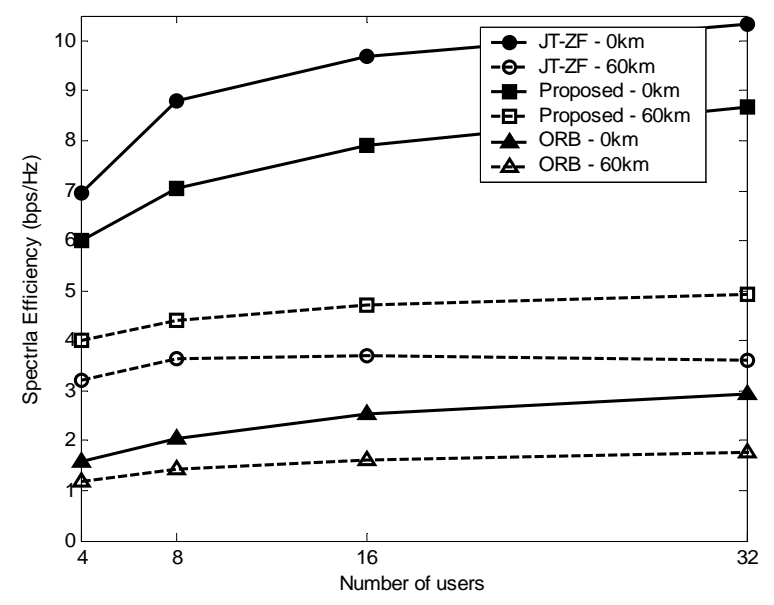

(b) $|\rho|=0.99$

Fig. 2. Performance of the proposed scheme according to the number of users.

the proposed scheme outperforms the orthogonal random beamforming. With the use of random beams in multiple BSs, the proposed scheme can achieve macro diversity gain as well as multi-user diversity gain. Note that the scheduled user to the first beam can not achieve an eigen-beamforming gain because of low spatial correlation, but it can still achieve co-phase reception gain. The proposed scheme can achieve noticeable eigen-beamforming and co-phase reception gain in high correlation channel environments (e.g., $|\rho|=0.99$ ), yielding performance comparable to the joint ZF beamforming scheme. When $|\rho|=0.99$, it can be seen that proposed scheme is less affected by the feedback delay. Despite of longer feedback delay, the proposed scheme can still achieve eigenbeamforming gain since the spatial correlation characteristic is remained. Thus, in the presence of high mobility and spatial correlation, the proposed scheme significantly outperforms the joint ZF beamforming scheme which suffers from severe CCI caused by channel mismatch.

\section{CONCLUSIONS}

We have proposed a BS cooperative transmission scheme working with partial CSI to reduce the implementation complexity and feedback signaling overhead. By utilizing three channel parameters, the proposed scheme can effectively achieve the beamforming and spatial multiplexing gain as well as the multi-user diversity gain. The performance of the proposed scheme has been analyzed and verified by computer simulation. The simulation results show that the proposed scheme is quite effective in high spatial correlation and manyuser environments, providing performance comparable to the joint ZF scheme that requires full CSI.

\section{REFERENCES}

[1]E. Telatar, "Capacity of multi-antenna Gaussian channels," AT\&T-Bell Technical Memorandum, 1995.

[2] G. J. Foschini and M. J. Gans, "On limits of wireless communications in a fading environment using multiple antennas," Wireless Personal Communications, vol. 6-3, pp. 311-335, 1998.

[3] S. Catreux, P. F. Driessen and L. J. Greenstein, "Simulation results for an interference-limited multiple-input multiple-output cellular system," IEEE Commun. Letters, vol. 4, no. 11, pp. 334-336, 2000.

[4] S. Das, H. Viswanathan and G. Rittenhouse, "Dynamic Load Balancing through Coordinated Scheduling in Packet Data," in Proc. IEEE INFOCOM, vol. 1, pp. 786-796, Mar. 2003.

[5]M. Costa, "Writing on dirty paper," IEEE Trans. Inform. Theory, vol. 29, no. 3, pp. 439-441, May. 1983.

[6] A. J. Goldsmith, S. A. Jafar, N. Jindal, and S. Vishwanath, "Capacity limits of MIMO Channels," IEEE J. Sel. Areas Commun, vol. 6, no. 3, pp. 311335, July 1998.

[7] S. A. Jafar, G. J. Foschini and A. J. Goldsmith, "PhantomNet: Exploring optimal multicellular multiple antenna systems," in Proc. IEEE VTC, vol. 1, pp. 261-265, Sept. 2002.

[8] S. Shamai and B. M. Zaidel, "Enhancing the cellular downlink capacity via co-processing at the transmitting end," in Proc. IEEE VTC, vol. 3, pp. 1745-1749, May 2001.

[9] G. Caire and S. Shamai, "On the achievable throughput of a multi-antenna Gaussian broadcast channel," IEEE Trans. Inform. Theory, vol. 49, pp. 1691-1706, July 2003.

[10] H. Zhang and H. Dai, "Co-channel Interference Mitigation and Cooperative Processing in Downlink Multicell Multiuser MIMO Networks," EURASIP J. Wireless Commun. and Networking, pp. 222-235, 2004

[11] M. K. Karakayali, G. J. Foschini, and R. A. Valenzuela, "Network coordination for spectrally efficient communications in cellular systems," Wireless Commun. Mag., vol. 3, no. 14, pp. 56-61, Aug. 2006.

[12] T. Yoo and A. J. Goldsmith, "Optimality of zero-forcing beamforming with multiuser diversity," in Proc. IEEE ICC, vol. 1, pp. 542-546, May 2005.

[13] M. Sharif and B. Hassibi, "On the capacity of MIMO broadcast channels with partial channel state information," IEEE Trans. Inform. Theory, vol. 51, pp. 506-522, Feb. 2005.

[14] A. Paulraj, R. Nabar and D. Gore, Introduction to Space-Time Wireless Communications, Cambridge, 2003.

[15] J. G. Proakis, Digital Communications, McGraw-Hill, 4-th ed., 2001.

[16] S. W. Halpern, "The effect of having unequal branch gains in practical predetection diversity systems for mobile radio," IEEE Trans. Veh. Technol., vol. VT-26, pp. 94-105, Feb. 1977.

[17] N. C. Beaulieu, "An infinite series for the computation of the complementary probability distribution function of a sum of independent random variables and its application to the sum of Rayleigh random variables," IEEE Trans. commun., vol. 26, pp. 1463-1474, Sept. 1990.

[18] J. Moon, J.-Y. Ko and Y.-H. Lee, "A framework design for the nextgeneration radio access system," IEEE J. Sel. Areas Commun., vol. 24, no. 3 , pp.554-564, Mar. 2006.

[19] W. C. Jakes, Microwave Mobile Communications, New York, NY, BEE Press, 1993. 